\title{
Human breast milk enhances intestinal mucosal barrier function and innate immunity in a pediatric human enteroid model
}

\author{
Gaelle Noel ${ }^{1 \# \&}$, Julie G. In ${ }^{2,3 \#}$, Jose M. Lemme-Dumit ${ }^{1 \#}$, Lauren R. DeVine ${ }^{4}$, Robert N. Cole ${ }^{4}$, \\ Anthony L. Guerrerio ${ }^{5}$, Olga Kovbasnjuk ${ }^{2,3^{*}}$ and Marcela F. Pasetti ${ }^{*}$
}

${ }^{1}$ Department of Pediatrics, Center for Vaccine Development and Global Health, University of Maryland School of Medicine, Baltimore, MD.

${ }^{2}$ Department of Internal Medicine, Division of Gastroenterology and Hepatology, University of New Mexico Health Science Center, Albuquerque, NM.

${ }^{3}$ Department of Medicine, Division of Gastroenterology and Hepatology, Johns Hopkins University School of Medicine, Baltimore, MD.

${ }^{4}$ Department of Biological Chemistry, Johns Hopkins Mass Spectrometry and Proteomics Facility, Johns Hopkins University School of Medicine, Baltimore, MD.

${ }^{5}$ Department of Pediatrics, Johns Hopkins University School of Medicine, Baltimore, MD.

\&Present address: Institut Pasteur, Center for Translational Science, 75015 Paris, France.

${ }^{*}$ Corresponding authors

Marcela F. Pasetti; 685 West Baltimore St. Room 480, Baltimore, MD 21201. Phone: (410) 8528957 E-mail: mpasetti@som.umaryland.edu

Olga Kobasnjuk; 1919 Lomas Blvd. NM 87106. Phone: (443) 791-8912 E-mail: okovbasnjuk@salud.unm.edu

\#These authors contributed equally to this work

Conflict of Interest: The authors have declared that no conflict of interests exist. 


\section{ABSTRACT}

2 Breastfeeding has been associated with long lasting health benefits. Nutrients and bioactive

3 components of human breast milk promote cell growth, immune development, and shield the

4 infant gut from insults and microbial threats. The molecular and cellular events involved in these

5 processes are ill defined. We have established human pediatric enteroids and interrogated

6 maternal milk's impact on epithelial cell maturation and function in comparison with commercial

7 infant formula. Colostrum applied apically to pediatric enteroid monolayers reduced ion

8 permeability, stimulated epithelial cell differentiation, and enhanced tight junction function by

9 upregulating occludin expression. Breast milk heightened the production of antimicrobial peptide

$10 \alpha$-defensin 5 by goblet and Paneth cells, and modulated cytokine production, which abolished

11 apical release of pro-inflammatory GM-CSF. These attributes were not found in commercial

12 infant formula. Epithelial cells exposed to breast milk elevated apical and intracellular plgR

13 expression and enabled maternal $\lg \mathrm{A}$ translocation. Proteomic data revealed a breast milk-

14 induced molecular pattern associated with tissue remodeling and homeostasis. Using a novel ex

15 vivo pediatric enteroid model, we have identified cellular and molecular pathways involved in

16 human milk-mediated improvement of human intestinal physiology and immunity. 


\section{INTRODUCTION}

19 The human gastrointestinal epithelium is a selective physical and chemical barrier that

20 separates the luminal content from the serosal compartment and inner host tissues (1). It

21 enables transport of electrolytes and nutrients, and provides a first line of defense against

22 pathogens by engaging innate and adaptive mucosal immune components (2). The intestinal

23 epithelium and associated mucosal immune environment progressively develop and mature

24 from early fetal stages through childhood by means of genetic and external signals $(3,4)$.

25 Human milk, rich in essential macronutrients, bioactive molecules (i.e., growth factors,

26 antimicrobial peptides, complex oligosaccharides), and immune components including

27 immunoglobulins, cytokines, and immune cells, supports tissue development and protects

28 infants against infectious agents (5). Human milk is also a source of and helps establish a

29 healthy microbiota in infants (6). Improvement of chronic and acute diseases (e.g., necrotizing

30 enterocolitis, inflammatory bowel diseases, and intestinal and pulmonary infections) has been

31 attributed to breastfeeding (7-10). Because of its countless benefits, breastfeeding has been

32 recommended at least during the first 6 months of life (11). Current knowledge of the health-

33 promoting benefits of human breast milk remains empiric or primarily descriptive, having been

34 derived from observational or epidemiologic studies. The cellular and molecular mechanisms

35 underlying the effects of maternal milk in the pediatric gut and physiologic pathways involved

36 remain ill characterized. One of the reasons for this gap in knowledge is the lack of reliable

37 models that could recapitulate the effect of human milk on the development and maintenance

38 of a healthy pediatric human gut and its origin in modulating systemic effects. Studies using

39 intestinal cancer cell lines including HT-29, T84, and Caco-2 cells or short-lived primary

40 epithelial cells obtained from animals fail to reproduce the normal physiological responses of

41 infant intestinal epithelium (12-15). Additionally, these immortalized cultures consist mainly of

42 enterocytes and lack intestinal segment- and age-specificity needed for study of the complex

43 multicellular and diverse composition of the human intestinal epithelium. 
44 In this study, we described the establishment of an ex vivo pediatric human enteroid model

45 derived from intestinal $\mathrm{Lgr5}^{+}$stem cells and a mechanistic interrogation of the effects of

46 human breast milk in the intestinal epithelium. Human intestinal enteroids (HIEs) recapitulate

47 the crypt-villus cell axis and the segment-specific physiology (duodenum, jejunum, ileum) of

48 the adult human small intestine $(16,17)$. Technical advantages of HIEs include their capacity

49 for long-term growth (years), which preserves donor genotype, and forming polarized

50 monolayers with easy access to apical and basolateral epithelial cell surfaces, which avoids

51 the cumbersome manipulation of 3D structures (18). Herein, we present a side-by-side

52 comparison of the molecular and cellular events affected by human milk vs. commercial infant

53 formula in human pediatric enteroids. Outcome analyses included pediatric intestinal tissue

54 morphology and maturation, ion and epithelial barrier permeability, antimicrobial and immune

55 functions, and epithelial cell secretome.

RESULTS

Pediatric and adult enteroid monolayers exhibit distinct cell morphology and maturation

features. To mechanistically interrogate the physiological effects of human breast milk in the

60 pediatric gut, differentiated enteroid monolayers were established from duodenal biopsies of

61 healthy 2- and 5-year-old children who underwent diagnostic endoscopy at The Johns Hopkins

62 Hospital, using methods previously described $(19,20)$; these monolayers are hereafter referred

63 to as 2PD and 5PD, respectively. The cell morphology, permeability and barrier integrity of the

64 pediatric monolayers were compared with those derived from adult duodenal tissue.

65 Differentiated (villus-like) enterocytes of pediatric origin were significantly shorter than their adult

66 counterparts as revealed by confocal microscopy images (Figure 1A) and epithelial cell height

67 measurement (Figure 1B). Analysis of the epithelial barrier function by transepithelial electrical

68 resistance (TER) revealed increased paracellular ion permeability in the pediatric- as compared

69 to the adult-derived monolayers (Figure $1 \mathrm{C}$ ). 
70 Human breast milk improves pediatric epithelial barrier function. We next examined the

71 effect of human breast milk (colostrum) on pediatric intestinal barrier function. Breast milk was

72 applied to the apical side of differentiated pediatric enteroid monolayers, and TER values were

73 monitored daily for $48 \mathrm{~h}$. Monolayers exposed to human breast milk exhibited higher TER values

74 as compared to non-treated controls (Figures 2A and B). A dose-response effect was observed,

75 with the $20 \%(\mathrm{v} / \mathrm{v})$ treatment resulting in higher TER values as compared to $2 \%(\mathrm{v} / \mathrm{v})$ (Figure

76 2A). This observation was consistent in multiple experiments using both lines; the $20 \%(\mathrm{v} / \mathrm{v})$

77 solution was therefore selected for subsequent experiments. We next compared ion

78 permeability of pediatric monolayers treated with human milk vs. commercial infant formula

79 (also resuspended at $20 \% \mathrm{w} / \mathrm{v}$ ). Human breast milk significantly and reliably increased TER

80 levels in both 2PD and 5PD monolayers as compared to non-treated controls and remained

81 elevated or further improved with prolonged exposure (Figures 2A and B). By contrast, ion

82 permeability was modestly affected by infant formula; TER values increased only in the 5PD

83 monolayer at $48 \mathrm{~h}$ of treatment (Figure 2B, right panel). In addition to transepithelial ion

84 permeability by TER, paracellular molecular permeability was examined by exposing breast

85 milk- and infant formula-treated pediatric monolayers to FITC-labelled 4kDa dextran for up to

$862 \mathrm{~h}$. No differences were observed in the amount of dextran recovered from the basolateral side

87 regardless of treatment (data not shown) confirming integrity of the epithelial barrier.

89 Human breast milk increases the expression of the tight junction (TJ) protein occludin.

90 Maternal milk enhancement of TER values prompted us to investigate its effect on expression of

91 TJ proteins, which seal the paracellular space of the intestinal epithelia and regulate passage of

92 ions and small molecules. Occludin, a transmembrane protein of the TJ complex was selected

93 for this analysis as crucial marker of epithelial differentiation and barrier function (21).

94 Immunofluorescent imaging revealed occludin on the cell perimeter of all monolayers,

95 regardless of treatment (Figure 3A). Strikingly, pediatric monolayers exposed to human milk 
96 exhibited a distinctive pattern of apical and condensed cytoplasmic vesicular expression of

97 occludin (Figure 3A) that markedly contrasted with the perimeter-only expression of monolayers

98 treated with infant formula. Quantitative analysis of the fluorescence intensity by confocal

99 imaging revealed superior occludin expression in both pediatric monolayers treated with human

100 breast milk as compared with monolayers treated with infant formula or untreated controls

101 (Figure 3B). Of the two enteroid lines, the 2PD was the higher and more consistent responder

102 (Figure 3B). Infant formula increased occludin expression modestly and occasionally, not

103 reaching significance above the non-treated controls (Figure 3B). The granular occludin

104 expression pattern induced by breast milk was observed not only in absorptive enterocytes,

105 visible by their prominent apical brush border, but also in cells lacking brush border, which are

106 typically secretory epithelial cell lineages such as Paneth cells, goblet cells, and

107 enteroendocrine cells (our HIE monolayers were not induced to express M cells). To identify the

108 specific cell types producing occludin, breast milk-treated monolayers were co-stained to detect

109 the presence of occludin as well as lysozyme, a marker for Paneth cells, trefoil factor 3 (TFF3),

110 a marker for goblet cells, and chromogranin A, a marker for enteroendocrine cells. Occludin

111 granular pattern co-localized with both lysozyme and TFF3, but not with chromogranin A marker

112 (Figure 3C). These results indicate that breast milk elevates occludin expression not only at the

113 TJ but also in the cytoplasm and apical membrane of absorptive enterocytes as well as in

114 Paneth cells and goblet cells.

116 Human milk increases epithelial cell expression of innate immune mediators. The

117 influence of breast milk on Paneth cell protein expression led us to examine its capacity to 118 enhance Paneth cell function, and in particular the production of antimicrobial peptides such as $119 \alpha$-defensin 5 (DEFA5), which helps maintain intestinal tolerance and homeostasis $(22,23)$.

120 DEFA5 fluorescence intensity was greatly increased in breast milk-treated pediatric monolayers 121 as compared to those treated with infant formula or non-treated controls (Figures $4 \mathrm{~A}$ and $\mathrm{B}$ ). 
122 Infant formula had no effect on DEFA5 expression. As expected, DEFA5 co-localized with

123 lysozyme ${ }^{+}$Paneth cells (Figure 4B). Surprisingly, a subpopulation of DEFA5-expressing cells

124 that lacked the lysozyme marker was observed in human milk-treated monolayers (Figure 4B).

125 Dual $\mathrm{DEFA5}^{+}$and $\mathrm{TFF}^{+}$fluorescent staining revealed co-localization of these two markers,

126 uncovering a breast milk-induced human goblet cell population with capacity to produce DEFA5

127 (Figure 4C).

128 We next examined the capacity of breast milk to modulate the production and secretion of 129 cytokines and chemokines typically produced by intestinal epithelial cells. IL-10, IFN- $\gamma$, TNF- $\alpha$,

130 IL-6, IL-8, MCP-1, and GM-CSF were measured in the apical and basolateral milieu of treated

131 and non-treated monolayers. IL-10 and IFN- $\gamma$ in all conditions were below limit of detection $(<0.7$

$132 \mathrm{pg})$. TNF- $\alpha$ and IL-6 were present at very low levels $(<1 \mathrm{pg})$ and below the limit of detection in

133 the non-treated controls, in both apical and basolateral compartments (data not shown). MCP-1,

134 GM-CSF, and IL-8 were detected in apical media and for the most part, levels increased over

135 time (Figures 4D-F). Treatment of pediatric monolayers with infant formula for $72 \mathrm{~h}$ resulted in a

136 marked increase of MCP-1 released apically as compared with non-treated monolayers. In

137 contrast, a trend of reduced MCP-1 production was observed upon treatment with human milk

138 (Figure 4D). GM-CSF was produced by untreated monolayers and by those treated with infant

139 formula. In fact, infant formula produced a slight - yet not statistically significant - upregulation

140 of GM-CSF at the $24 \mathrm{~h}$ time point (Figure 4E). Conversely, apical GM-CSF secretion was

141 abolished when monolayers were treated with human milk, at both time points tested (Figure

142 4E). Apical release of IL-8 remained unaffected by treatment (Figure 4F). Basolateral secretion

143 of MCP-1, GM-CSF, and IL-8 was not influenced by treatment either (data not shown). A

144 principal component analysis (PCA) was conducted combing $24 \mathrm{~h}$ outcomes described above to

145 visualize, in aggregate, the impact of breast milk and infant formula on epithelial cell physiology

146 (the $24 \mathrm{~h}$ time point was selected because it allowed for a complete dataset for all treatments). 
147 Monolayers untreated or exposed to infant formula clustered together and were largely distant

148 from those exposed to breast milk by principal component 1 (Figure 4G). Breast milk treatment

149 was associated with biomarkers of enhanced barrier function (DEFA5, occludin, and TER), 150 whereas infant formula was linked to synthesis of pro-inflammatory cytokines (IL-8, MCP-1, and 151 GM-CSF) (Figure 4G).

152

153 Human milk sigA translocates across pediatric enteroid monolayers. Breast milk contains

154 a variety of immune mediators, including antibodies that shield immunologically naïve infants 155 from health threats. Maternal immunoglobulins, in particular slgA, support infant immune 156 development and regulation, enacting long lasting benefits. Early colostrum has high levels of 157 maternal $\operatorname{sig} A$ and $\lg G$, and hence our system enabled us to investigate their interaction with 158 pediatric intestinal epithelial cells. Both 2PD and 5PD monolayers expressed secretory 159 component (SC) of the polymeric immunoglobulin receptor (plgR), which mediates $\lg A$ 160 translocation across the intestinal epithelium as well as the neonatal Fc receptor (FcRn), 161 responsible for transepithelial lgG transpot as shown by immunoblotting (Figures $5 \mathrm{~A}$ and $\mathrm{B}$ ).

162 Confocal microscopy images revealed a diffuse cytoplasmic SC-plgR expression in the non163 treated controls, whereas epithelial cells exposed to breast milk exhibited not only intracellular 164 but also basolateral and dense apical SC-plgR expression (Figure 5A). Enhanced SC-plgR 165 expression in pediatric monolayers treated with breast milk was confirmed by immunoblotting 166 (Figure 5C). Soluble SC-plgR was detected in milk alone but not in infant formula (Figure 5C).

167 We next compared apical to basolateral slgA and $\lg G$ translocation in monolayers treated with 168 breast milk vs. non-treated controls. Both slgA and IgG were detected on basolateral side of 169 milk-exposed epithelial cells; slgA levels were significantly higher than those of the non-treated 170 controls (Figure 5D). 


\section{Breast milk-induced protein upregulation and basolateral secretion by pediatric epithelial}

173 cells. The intestinal epithelium communicates with underlying tissues via secretion of nutrients,

174 growth factors, cytokines, and regulatory peptides. Gut-derived molecules secreted to the

175 basolateral compartment have the potential to disseminate systemically and act on remote 176 tissues, exacting distant modulatory functions. To identify breast milk-induced molecules of

177 intestinal origin that may have a wider (and possibly systemic) impact in vivo, we examined

178 proteins secreted into the basolateral compartment of milk-exposed monolayers. Over 6000

179 proteins were identified by a proteomic analysis. To select the differentially expressed proteins

180 from a total of 392 secreted proteins (with high false discovery rate), we applied the cutoffs:

181 adjusted $p$-value $\leq 0.05$ and $\log _{2}$ fold change at \pm 0.68 . A total of 61 proteins had increased

182 abundance in the breast milk-treated enteroids, whereas 21 were increased in the non-treated

183 control (Figure 6A).

184 Proteins derived from human milk were found in the basolateral compartment of breast milk-

185 treated monolayers, indicating apical to basolateral transepithelial translocation.

186 In addition, we observed increased levels of proteins related to mucosal protection and repair

187 (e.g., TFF1-3, lysozyme C, amyloid-like protein), epithelial cell markers (e.g., EpCAM), growth

188 factors (e.g., insulin-like growth factor-binding protein [IGFBP], fibroblast growth factor binding 189 protein [FGFBP]), extracellular matrix remodeling proteins (e.g., metalloproteinase inhibitor 190 proteins, basement membrane-specific heparan sulfate proteoglycan core protein) and cofactor

191 carrier protein (e.g., transcobalamin 2) in the human breast milk-treated monolayers (Figure

192 6A). In contrast, the non-treated monolayers exhibited increased expression of the 193 apolipoprotein family, and annexin $\mathrm{V}$ (Figure 6A). The interactions among proteins with

194 increased abundance in the breast milk-treated enteroids were examined using the STRING 195 v11.0 database. The analysis revealed a significant protein-protein interaction $\left(\mathrm{p}-\mathrm{value}<1.0^{-16}\right)$

196 among 57 of them (228 edges), whereas 4 proteins showed no interactions within the network 197 (Figure 6B). These results indicate that most of the proteins secreted by milk-exposed enteroids 
198 do not act as independent entities but can deploy biological activity by either transient or stable

199 association. A functional enrichment analysis was then performed utilizing the PANTHER and

200 AMIGO2 classification database system to highlight the gene ontology (GO) terms annotated for

201 cellular component, molecular function, and biological processes enriched within these protein

202 sets (Figure 6C). The majority of the proteins were associated with the extracellular

203 compartment (24.3\%; GO:0044421, GO:0005576) as well as within the cell (12.1\%;

204 GO:0044464, GO:0005623) as constitutive protein with cytoplasmic or plasma membrane

205 localization. The main molecular function identified was binding (51.4\%; GO:0005488) followed

206 by enzyme activity (28.6\%; GO:0003824). In addition, these protein sets participate in multiple

207 biological processes, including cell physiology, response to stimulus, metabolic functions, cell

208 growth and maintenance, and immunity (Figure 6C).

210 DISCUSSION

211 Human breast milk is a rich source of nutrients and bioactive components that promote infant

212 growth and immune development. In this work, using an ex vivo pediatric intestinal stem cell-

213 derived human enteroid model, we have identified distinct protein synthesized and cellular

214 functions modulated by human breast milk. HIEs represent a cutting-edge technology that

215 recapitulates the structural and functional features of the human gastrointestinal tissue. They

216 have been used to interrogate gut physiology, host responses to microbes, drug activity, and

217 cell-to-cell communication (24-29). A side-by-side comparison of pediatric- vs. adult-derived

218 duodenal HIE monolayers revealed age-associated differences with the former exhibiting

219 shorter columnar epithelial cells and reduced TER, consistent with a less mature epithelial cell

220 phenotype. Reduced enterocyte height has been reported in duodenal biopsies of infants, as

221 compared to adult subjects (30). Together, these results suggest that intestinal epithelial cell

222 development continues through childhood. They also demonstrate that age-specific cell

223 morphology is preserved in the HIEs. 
224 Several unique molecular events associated with human milk improvement of pediatric intestinal

225 health were observed. The first was the ability of breast milk (colostrum) to enhance epithelial

226 barrier function by reducing ion permeability and upregulating expression of the TJ complex

227 regulator occludin. The breast milk-treated monolayers exhibited an unusual pattern of

228 upregulated occludin protein expression. Occludin was detected not only at the (expected)

229 intercellular junctions but also on the apical plasma membranes of absorptive enterocytes as

230 well as Paneth and goblet cells. Condensed occludin-containing vesicles were spread

231 intracellularly. Apical occludin localization has been reported recently in mouse organoids,

232 primarily in intestinal stem cells and Paneth cells, and less abundantly in enterocytes and goblet

233 cells, and its presence associated with reduced paracellular permeability (31). A regulatory

234 mechanism that involves recruitment of occludin contained in cytoplasmic vesicles or in the

235 apical plasma membrane (via differential phosphorylation) for TJ formation has been proposed

236 (32); under this model, the extra junctional localization may represent protein reservoirs that

237 enable prompt TJ formation required by dynamic metabolic and physiological processes. To the

238 best of our knowledge, this is the first demonstration of apical and cytoplasmic multi-lamellar

239 occludin expression by human pediatric intestinal cells upregulated in response to breast milk.

240 A second key observation was the capacity of human milk to substantially increase production

241 of human DEFA5, a peptide that contributes to innate host defense against enteropathogens

242 and promotes intestinal homeostasis by limiting inflammation and microbial translocation (22,

243 33, 34). DEFA5 was produced not only by Paneth cells (the typical producers of antimicrobial

244 molecules) but also by mucus-producing goblet cells. Production of DEFA5 by intestinal villous

$245 \mathrm{TFF3}^{+}$(goblet cells) but not lysozyme ${ }^{+}$cells has been documented in human ileal biopsies (35).

246 Goblet and Paneth cells derive from a common secretory cell progenitor under the regulation of

247 ETS transcription factor Spdef (36). Lgr5 ${ }^{+}$stem cells and Paneth cells are abundant in crypt-like,

248 non-differentiated HIEs. The lifespan of Paneth cells in enteroids is approximately 30 days,

249 regardless of differentiation, as was previously shown in adult differentiated 3D enteroids (16). 
By contrast, the expression of DEFA5 in TFF3 ${ }^{+}$goblet cells, which mark the differentiated small

251 intestinal epithelium, is a new finding and may reflect a differentiating cell lineage stage

252 prompted by breast milk-derived growth factors. The heightening production of TJ proteins and

253 antimicrobial products induced by breast milk (but not infant formula) is consistent with the

254 reported improved epithelial barrier of infants fed with breast milk over those fed by formula as

255 determined by reduced ratio of lactulose-to-mannitol in urine (37).

256 A third important observation was the immune modulation associated with human milk treatment

257 of pediatric epithelial cells. While infant formula increased the production of pro-inflammatory

258 cytokines MCP-1 and GM-CSF, breast milk reduced MCP-1 levels and totally suppressed apical

259 release of GM-CSF. Gut inflammatory diseases such as intestinal bowel disease and celiac

260 disease coincide with elevated MCP-1 and GM-CSF in duodenal biopsies (38).

261 IL-8, an epithelial cell-derived neutrophil chemoattractant was produced by the pediatric

262 intestinal epithelium. Although not overtly affected by treatment, IL-8 was associated with

263 exposure to infant formula as shown by PCA analysis of early time-point outcomes. These

264 results are consistent with the anti-inflammatory properties of human milk, which, in the pediatric

265 gut are deployed by reducing or abolishing steady state levels of signals that may activate or

266 recruit phagocytic cells and enhance pro-inflammatory cytokines (i.e., GM-CSF and MCP-1)

267 (39).

268 Different from adult HIEs, the pediatric HIEs did not produce substantial levels of TGF- $\beta 1$, IFN- $\gamma$,

269 IL-6, and TNF- $\alpha$ (19); these findings suggest that beyond the immune modulation of maternal

270 milk, the pediatric intestinal epithelium is intrinsically programmed to silence signals that trigger

271 inflammatory processes.

272 Human milk's composition is complex and dynamic, and encompasses a vast diversity of

273 soluble components that act as prebiotics, antiadhesives, antimicrobials, as well as molecules

274 that affect cellular physiology, shield the host from inflammatory and pathogenic insults (40), 
275 and promote healthy gut development. Bioactive components with attributed anti-inflammatory 276 and homeostatic function in human milk include IL-10, TGF- $\beta$, antioxidants, and enzymes such 277 as lysozyme, glutathione peroxidase, and catalase (41). Additionally, human milk provides a 278 variety of growth factors and tissue development/remodeling agents (42); proteomic analyses of 279 human breast milk have been reported elsewhere $(43,44)$. We showed herein that many of 280 these milk-derived components gain access to the subcellular space (see below). The exact 281 molecules that trigger the effects described above and operatives, whether they work alone or in 282 a synergistic/complementary manner, remain to be elucidated.

283 Maternal milk-derived slgA provides an additional protective immune layer that excludes, 284 neutralizes, and prevents microbial attachment to host cells (45). Mucosal dimeric IgA binds to 285 plgR on the basolateral surface of the epithelial cell membrane, is transported intracellularly and 286 released at the apical surface, carrying a small portion of the plgR-binding domain (46), the SC.

287 Similar mechanism allows for $\lg$ epithelial transport, whereas $\lg$ employs the FcRn to 288 bidirectionally cross epithelial tissues (47). Maternal antibodies provide antigen-specific 289 defenses, support homeostasis, and promote infant immune development. In animal models, 290 breast milk slgA conferred long lasting benefits that included maintenance of a healthy 291 microbiota and regulation of epithelial cell gene expression (48). A fourth relevant finding was 292 the visualization of plgR in the apical and basolateral membrane of breast milk-treated 293 enterocytes. Breast milk itself contained an abundance of soluble SC-plgR, but none was 294 detected in commercial infant formula. The soluble SC-plgR in maternal milk likely originates 295 from maternal cellular debris. Free SC in human milk can bind enteric pathogens and toxins, 296 and thus boosts non-specific host defenses (49) in the infant gut. We detected apical-to-basal 297 slgA transport in the maternal milk-exposed pediatric monolayers. This process supports 298 intracellular pathogen neutralization and delivery of luminal antigens to lamina propria dendritic 299 cells to induce tolerance or subepithelial phagocytic cells to imprint antigen specific immunity 
300 (50). FcRn detection in the pediatric tissue confirms expression of this receptor beyond infancy.

301 Others have reported FcRn being expressed in human intestinal epithelial cells $(51,52)$.

302 We were unable to detect translocation of maternal $\lg \mathrm{G}$, despite this process being documented 303 in animal models and cell lines (53). The variable localization of FcRn and pH requirements may

304 restrict apical-to-basolateral transport while basolateral-to-apical appears to be more prevalent

305 (54). Studies of FcRn distribution, IgG interaction and IgG immune complex translocation in 306 pediatric HIEs are ongoing.

307 Beyond promoting a healthy gut, multiple and far reaching benefits have been attributed to 308 human milk, including prevention of respiratory diseases, immune fitness, cognitive capacity, 309 and overall physiological well-being (55) that endure into adolescence. Breast milk products 310 released to the basal side of the epithelium could, conceivably, distribute systemically and 311 thereby mediate long distant effects. Our proteomic analysis of breast milk-treated monolayers 312 revealed a variety of molecules, some unique to breast milk, such as $\alpha$-lactalbumin, $\beta$-casein, 313 and prolactin, which had evidently translocated across the monolayers, and others that were 314 produced by the milk-exposed pediatric intestinal cells. For the latter, a complex network of 315 interacting biomolecules was revealed, with diverse functions including those affecting growth 316 factors, immune and antimicrobial activity, tissue structure, and homeostasis, which confirms 317 the broad and pleotropic nature of the processes affected by breast milk. The epithelial 318 translocation of milk-derived proteins might have been facilitated by endocytosis of intact 319 (undigested) molecules in our model. These proteins have health benefits by themselves. Milk $320 \alpha$-lactalbumin, for example, shields soluble CD14 (sCD14) from proteolytic degradation (56), 321 and sCD14 can bind lipopolysaccharide (LPS) and prevent inflammation and injury caused by 322 soluble LPS or LPS-bearing organisms. $\beta$-casein is an immune modulator that regulates cell 323 recruitment, ameliorates inflammation, and stimulates mucus production (57). Prolactin is a 324 pleiotropic hormone that stimulates production of maternal milk. Expected benefits for the infant, 
325 based on animal studies, include reduction of anxiety and stress and neurogenesis (58). In 326 addition, osteopontin prevents inflammation and epithelial damage in mouse DSS-colitis model 327 (59).

328 A variety of breast milk-upregulated tissue-derived proteins were identified, including the TFF 329 family, which maintains and restores gut mucosal homeostasis and regulates complement 330 activation via decay-accelerating factor, DAF (60); the amyloid-like protein, which participates in 331 intestinal metabolic processes and modulates expression of MHC class I molecules $(61,62)$; 332 and, insulin growth factor binding protein, fibroblast growth factor, basement membrane-specific 333 heparan sulfate protein, and metalloproteinase inhibitor - all of which contribute to epithelial cell 334 growth, tissue remodeling, and barrier integrity (63-65). Other secreted proteins included 335 transcobalamin 2, which facilitates the transport of vitamin B12 within the organs (66) and 336 epithelial cell adhesion molecule (EpCAM), which localizes in the basal cell membrane and 337 facilitates cell-to-cell interaction and proliferation (67). Complement proteins (C3 and C4) were 338 also present in the basal media from breast milk-treated enteroids; C4 participates in 339 complement activation via the classical and lectin pathway, whereas C3 is a converging 340 substrate for all activating pathways; C3 cleavage into C3a and C3b, along with C5 cleavage, 341 trigger the rest of the complement cascade. C3, C4, and other complement components are 342 present in human breast milk $(43,44)$. Likewise, human intestinal epithelial cells produce 343 complement proteins $(68,69)$. The origin of the complement proteins we identified is unclear.

344 We surmise they derive from breast milk because synthesis of complement proteins by the 345 intestinal epithelial cells reportedly requires pro-inflammatory signals (downregulated by breast 346 milk in our system) (70). Nonetheless, the fact that maternal complement molecules would 347 trespass the pediatric epithelium is intriguing. Regardless of their source, complement can boost 348 infant mucosal protective mechanisms (71). 
349 Bovine colostrum has been shown to influence the proteome of HT-29 cells as well as epithelial 350 cell glycosylation (72). We show, for the first time, that human milk influences the synthesis of

351 multiple mediators of metabolic and physiologic functions that act locally or systemically.

352 In summary, using a novel ex vivo pediatric HIE, several mechanisms associated with breast 353 milk were identified that improve intestinal health: 1) cell differentiation and strengthening of the 354 pediatric intestinal barrier by reduction of permeability and upregulation of TJ occludin with a 355 unique expression pattern; 2) boosting of innate immunity by enhancing production of 356 antimicrobial DEFA5 by Paneth and goblet cells; 3) immune modulation and passive 357 immunization by increased production of $\mathrm{plgR}$ and translocation of luminal slgA; 4) reduction of 358 pro-inflammatory cytokines; 5) translocation of breast milk proteins with anti-inflammatory and 359 anti-microbial properties; and 6) expression of proteins responsible for tissue remodeling and 360 mucosal homeostasis.

\section{Methods}

363 Study approval. Protocols for recruitment of human participants, obtaining informed consent,

364 collecting and de-identifying biopsy samples were approved by the Johns Hopkins University 365 School of Medicine (JHU SOM) Institutional Review Board (IRB) NA 00038329. Procedures for 366 recruitment of mothers around delivery, obtaining informed consent and collection and de367 identification of breast milk were approved under University of Maryland School of Medicine IRB 368 HP-00065842.

370 Generation of enteroid monolayers. Duodenal biopsies were obtained from 5 healthy

371 individuals, two pediatrics (ages 2, 5) and three adults (ages 25, 27, and 81 years) through 372 endoscopy or surgical procedure. Enteroids were generated from $\mathrm{Lgr5}^{+}$intestinal crypts 373 embedded in Matrigel (Corning, USA) in 24-well plates, as previously described (73). Enteroids 374 were expanded in growth factor-enriched media containing Wnt3A, Rspo-1, and Noggin (18, 
375 19). Multiple enteroid cultures were harvested with Culturex Organoid Harvesting Solution

376 (Trevigen, USA), fragmented and re-suspended in expansion media and seeded (100 $\mu$ l) on the

377 inner surface of $0.4 \mu \mathrm{m}$ Transwell inserts (Corning, USA), pre-coated with human collagen IV

378 (Sigma-Aldrich, USA), and $600 \mu$ of expansion media was added to the receiver plate well.

379 Media was replenished every other day (20). Enteroid monolayer confluency was monitored by

380 measuring TER, as previously described (20). Upon reaching confluency, monolayers were

381 differentiated in media (DFM) free of Wnt3A and Rspo-1 for 5 days (20). All cultures were

382 maintained at $37^{\circ} \mathrm{C}$ and $5 \% \mathrm{CO}_{2}$.

384 Breast milk preparation and monolayer treatment. Human colostrum was obtained from

385 women 0-3 days post-delivery. Commercial infant formula powder (Similac $\circledast$ Advance $®$ Abbot

386 Nutrition) was resuspended in sterile distilled water following manufacturer's instructions. Both

387 human breast milk and infant formula suspensions were centrifuged twice (10 min each) at

$3883,000 \mathrm{~g}$. The soluble fractions were extracted, aliquoted, and stored at $-80^{\circ} \mathrm{C}$ until use. Enteroid

389 monolayers were treated apically with $100 \mu$ of human milk or infant formula diluted 2 or $20 \%$ in

390 DFM. Non-treated controls were treated with $100 \mu$ of DFM. TER was monitored daily while

391 conducting experiments to ensure monolayer integrity.

393 Dextran permeability assay. FITC-labelled 4 kDa dextran (Millipore Sigma, St. Louis, MO;

$3940.01 \% \mathrm{w} / \mathrm{v}$ in DFM) was added to the apical side of enteroid monolayers pre-treated with $20 \%$ of

395 human milk or infant formula. Regular DFM $(600 \mu \mathrm{l})$ was added to the basolateral side.

396 Basolateral media $(100 \mu \mathrm{l})$ was sampled at $30 \mathrm{~min}, 1$ and $2 \mathrm{~h}$, and FITC-dextran content was

397 measured by fluorescence intensity using an EnVision Multilabel Plate Reader (PerkinElmer,

398 Waltham, MA). Sampled volume was replenished with fresh DFM. 
Immunofluorescence staining and confocal imaging. Enteroid monolayers were fixed for 40

401 min in 4\% paraformaldehyde (Electron Microscopy Sciences, USA), washed with PBS for 10

402 min, permeabilized and blocked for $1 \mathrm{~h}$ with PBS containing $15 \%$ fetal bovine serum, $2 \%$ BSA,

403 and $0.1 \%$ saponin, all at room temperature (RT). After washing with PBS, monolayers were

404 incubated overnight at $4^{\circ} \mathrm{C}$ with primary antibodies (diluted 1:100 in PBS). The following primary

405 antibodies $(\mathrm{Ab})$ were used: occludin (mouse monoclonal [mAb], clone OC-3F10, Thermo Fisher

406 Scientific), TFF3 (rabbit polyclonal [pAb], Millipore Sigma), lysozyme EC 3.2.1.17 (rabbit pAb,

407 Dako), DEFA5 (mouse mAb, clone 8C8, Millipore Sigma), and SC-166 (mouse mAb provided by

408 Dr. A. Hubbard, Johns Hopkins University School of Medicine). Stained monolayers were

409 washed with PBS (3 times, 10 min each) and incubated with secondary antibodies (diluted

$4101: 100$ in PBS) for $1 \mathrm{~h}$ at RT. Secondary antibodies included goat anti-mouse Alexa Fluor-488 or

$411-568$, and goat anti-rabbit Alexa Fluor-488 or -568 (all Thermo Fisher Scientific). F-actin was

412 detected by phalloidin Alexa Fluor-633, -647, or -568 (1:100; Thermo Fisher Scientific). Hoechst

413 for nuclear/DNA labeling (Thermo Fisher Scientific) was used diluted 1:1000 in PBS. After

414 incubation, cells were washed as described above, and mounted in FluorSave reagent (Millipore

415 Sigma). Confocal images were taken using an LSM-510 META laser scanning confocal

416 microscope (Zeiss, Germany) and ZEN 2012 imaging software (Zeiss) or BZ-X700 fluorescence

417 microscope (Keyence, Japan) available through the Fluorescence Imaging Core of the Hopkins

418 Basic Research Digestive Disease Development Center. For qualitative analysis, image settings

419 were adjusted to optimize the signal. For quantitative analysis, the same settings were used

420 across the samples, and protein-of-interest average intensity fluorescence was analyzed using

421 MetaMorph software (Molecular Devices, CA).

423 Protein extraction, immunoblotting, and proteomic analysis. Enteroid monolayers were 424 lysed in cold lysis buffer (60 mM HEPES pH 7.4, 150 mM KCl, 5 mM Na3EDTA, 5 mM EGTA, 1 $425 \mathrm{mM}$ Na3VO4, $50 \mathrm{mM} \mathrm{NaF}, 2 \%$ SDS) supplemented with 1:100 of protease inhibitor cocktail 
426 (P8340, Millipore Sigma). Lysis buffer was applied to the apical surface, and cells were scraped

427 and sonicated on ice ( 3 times at 10 sec pulses each time using $30 \%$ energy input). The lysates

428 were centrifuged $10 \mathrm{~min}$ at $14000 \mathrm{rpm}$ at $4^{\circ} \mathrm{C}$, and the supernatant containing soluble and

429 membrane proteins was collected. Total protein concentration was determined using a DC

430 protein assay (Bio-Rad, CA). Proteins were separated on Novex Wedgewell 4-20\% gradient

431 Tris-glycine gels (Life Technologies, CA) and transferred to nitrocellulose membranes. The

432 following primary antibodies were used for immunoblotting: polyclonal rabbit anti-plgR (Abcam),

433 monoclonal mouse anti-SC-166, and polyclonal rabbit anti-FcRn (Novus Biologicals) - all at a

434 1:250 dilution, and mouse monoclonal anti-GAPDH (clone 6C5, Abcam) at 1:1000 dilution.

435 Secondary antibodies included goat anti-mouse Alexa Fluor-488 or -568 and goat anti-rabbit

436 Alexa Fluor-488 or -568 (Thermo Fisher Scientific). Western blots were processed using the

437 iBind Flex device (Life Technologies, Carlsbad, CA) and then imaged on an Odyssey CLx

438 imager (LI-COR, Lincoln, NE). Proteomic analysis was conducted on basolateral media from

439 pediatric monolayers treated with human milk $(n=3)$ and non-treated control $(n=2)$ through the

440 Mass Spectrometry and Proteomics Facility, Johns Hopkins University School of Medicine.

442 Cytokines/chemokines. Cytokines and chemokines were quantified using commercial

443 electrochemiluminescence microarray kits (Meso Scale Diagnostic, Rockville, MD) following the

444 manufacturer's instructions. MCP-1, GM-CSF, and IL-8 levels were reported as the amount

445 contained in the total volume of culture supernatant collected from the apical and basolateral

446 side of the monolayers.

448 Statistics. Statistical significances were calculated using the Student's $t$-test to compare two

449 groups, or one-way-ANOVA with Šidák's or Tukey's post-test as appropriate among more than

450 two groups. PCA was performed by selecting PC with eigenvalues greater than 1.0 (Kaiser 
451 rule). Plots and statistical tests were performed using Prism software v9 (GraphPad, San Diego,

$452 \mathrm{CA})$. Differences were considered statistically significant at $p$-value $\leq 0.05$.

\section{Author contributions}

455 GN, JGI, and JML-D conducted experiments and analyzed data; JML-D compiled final figures;

456 LD and RC conducted proteomics analysis; AG obtained pediatric biopsies; OK and MFP

457 conceptualized the study, secured funding, designed experiments and data analysis. All authors

458 contributed to the writing and editing of the manuscript.

\section{Acknowledgements}

461 This work was supported by a Grand Challenge Exploration award (Bill and Melinda Gates

462 Foundation) OPP 1118529 and in part, by NIH grants R01Al117734 (to MFP), P01 Al125181 (to

463 MFP and OK) and K01 DK106323 (JGI). The authors acknowledge the Integrated Physiology

464 and Imaging Cores of the Hopkins Digestive Disease Basic and Translational Research Core 465 Center (P30 DK089502) and the Johns Hopkins Mass Spectrometry and Proteomics Core. 


\section{REFERENCES}

$467 \quad$ 1. Zihni C, et al. Tight junctions: from simple barriers to multifunctional molecular gates. Nat Rev Mol Cell Biol. 2016;17(9):564-80. Peterson LW, and Artis D. Intestinal epithelial cells: regulators of barrier function and immune homeostasis. Nat Rev Immunol. 2014;14(3):141-53. Torow N, et al. Neonatal mucosal immunology. Mucosal Immunol. 2017;10(1):5-17.

4. Stras SF, et al. Maturation of the Human Intestinal Immune System Occurs Early in Fetal Development. Dev Cell. 2019;51(3):357-73 e5.

5. Turfkruyer $M$, and Verhasselt V. Breast milk and its impact on maturation of the neonatal immune system. Curr Opin Infect Dis. 2015;28(3):199-206.

6. Ballard O, and Morrow AL. Human milk composition: nutrients and bioactive factors. Pediatr Clin North Am. 2013;60(1):49-74.

7. Bode L. Human Milk Oligosaccharides in the Prevention of Necrotizing Enterocolitis: A Journey From in vitro and in vivo Models to Mother-Infant Cohort Studies. Front Pediatr. 2018;6:385.

8. Jantscher-Krenn E, et al. The human milk oligosaccharide disialyllacto-N-tetraose prevents necrotising enterocolitis in neonatal rats. Gut. 2012;61(10):1417-25.

9. Barclay AR, et al. Systematic review: the role of breastfeeding in the development of pediatric inflammatory bowel disease. J Pediatr. 2009;155(3):421-6.

10. Oddy WH. Breastfeeding, Childhood Asthma, and Allergic Disease. Ann Nutr Metab. 2017;70 Suppl 2:26-36.

11. World Health Organization. WHO Recommendations on Postnatal Care of the Mother and Newborn. Geneva; 2013.

12. Sun D, et al. Comparison of human duodenum and Caco-2 gene expression profiles for 12,000 gene sequences tags and correlation with permeability of 26 drugs. Pharm Res. 2002;19(10):1400-16.

13. Drummond CG, et al. Enteroviruses infect human enteroids and induce antiviral signaling in a cell lineage-specific manner. Proc Natl Acad Sci U S A. 2017;114(7):1672-7.

14. Lin $S$, et al. Comparison of the transcriptional landscapes between human and mouse tissues. Proc Natl Acad Sci U S A. 2014;111(48):17224-9.

15. Pulendran $\mathrm{B}$, and Davis MM. The science and medicine of human immunology. Science. 2020;369(6511).

16. Sato T, et al. Single Lgr5 stem cells build crypt-villus structures in vitro without a mesenchymal niche. Nature. 2009;459(7244):262-5.

17. Zachos NC, et al. Human Enteroids/Colonoids and Intestinal Organoids Functionally Recapitulate Normal Intestinal Physiology and Pathophysiology. J Biol Chem. 2016;291(8):3759-66.

18. In JG, et al. Human colonoid monolayers to study interactions between pathogens, commensals, and host intestinal epithelium. J Vis Exp. 2019(146).

19. Noel G, et al. A primary human macrophage-enteroid co-culture model to investigate mucosal gut physiology and host-pathogen interactions. Sci Rep. 2017;7:45270.

20. Staab JF, et al. Co-Culture System of Human Enteroids/Colonoids with Innate Immune Cells. Curr Protoc Immunol. 2020;131(1):e113. 
21. Al-Sadi $\mathrm{R}$, et al. Occludin regulates macromolecule flux across the intestinal epithelial tight junction barrier. Am J Physiol Gastrointest Liver Physiol. 2011;300(6):G1054-64.

22. Bevins CL, and Salzman NH. Paneth cells, antimicrobial peptides and maintenance of intestinal homeostasis. Nat Rev Microbiol. 2011;9(5):356-68.

23. Sankaran-Walters S, et al. Guardians of the Gut: Enteric Defensins. Front Microbiol. 2017;8:647.

24. In JG, et al. Epithelial WNT2B and Desert Hedgehog Are Necessary for Human Colonoid Regeneration after Bacterial Cytotoxin Injury. iScience. 2020;23(10):101618.

25. Liu L, et al. Mucus layer modeling of human colonoids during infection with enteroaggragative E. coli. Sci Rep. 2020;10(1):10533.

26. Co JY, et al. Controlling epithelial polarity: A human enteroid model for host-pathogen interactions. Cell Rep. 2019;26(9):2509-20.e4.

27. King AJ, et al. Inhibition of sodium/hydrogen exchanger 3 in the gastrointestinal tract by tenapanor reduces paracellular phosphate permeability. Sci Transl Med. 2018;10(456).

28. Lin SC, et al. Human norovirus exhibits strain-specific sensitivity to host interferon pathways in human intestinal enteroids. Proc Natl Acad Sci U S A. 2020;117(38):2378293.

29. Chang-Graham AL, et al. Rotavirus induces intercellular calcium waves through ADP signaling. Science. 2020;370(6519).

30. Thompson FM, et al. Epithelial growth of the small intestine in human infants. $J$ Pediatr Gastroenterol Nutr. 1998;26(5):506-12.

31. Pearce SC, et al. Marked differences in tight junction composition and macromolecular permeability among different intestinal cell types. BMC Biol. 2018;16(1):19.

32. Wong V. Phosphorylation of occludin correlates with occludin localization and function at the tight junction. Am J Physiol. 1997;273(6):C1859-67.

33. Salzman $\mathrm{NH}$, et al. Enteric defensins are essential regulators of intestinal microbial ecology. Nat Immunol. 2010;11(1):76-83.

34. Ehmann D, et al. Paneth cell alpha-defensins HD-5 and HD-6 display differential degradation into active antimicrobial fragments. Proc Natl Acad Sci U S A. 2019;116(9):3746-51.

35. Cunliffe RN, et al. Human defensin 5 is stored in precursor form in normal Paneth cells and is expressed by some villous epithelial cells and by metaplastic Paneth cells in the colon in inflammatory bowel disease. Gut. 2001;48(2):176-85.

36. Gregorieff A, et al. The ets-domain transcription factor Spdef promotes maturation of goblet and paneth cells in the intestinal epithelium. Gastroenterology. 2009;137(4):1333-45 e1-3.

37. Catassi $C$, et al. Intestinal permeability changes during the first month: effect of natural versus artificial feeding. J Pediatr Gastroenterol Nutr. 1995;21(4):383-6.

38. Di Sabatino A, et al. Innate and adaptive immunity in self-reported nonceliac gluten sensitivity versus celiac disease. Dig Liver Dis. 2016;48(7):745-52.

39. Hamilton JA. GM-CSF in inflammation. J Exp Med. 2020;217(1).

40. Bode L. The functional biology of human milk oligosaccharides. Early Hum Dev. 2015;91(11):619-22. 
552 41. Cacho NT, and Lawrence RM. Innate Immunity and Breast Milk. Front Immunol. 2017;8:584.

42. Ogra PL. Immunology of Human Milk and Lactation: Historical Overview. Nestle Nutr Inst Workshop Ser. 2020;94:11-26.

43. Zhu J, and Dingess KA. The Functional Power of the Human Milk Proteome. Nutrients. $2019 ; 11(8)$.

44. Gao X, et al. Temporal changes in milk proteomes reveal developing milk functions. J Proteome Res. 2012;11(7):3897-907.

45. Cerutti $A$, and Rescigno $M$. The biology of intestinal immunoglobulin A responses. Immunity. 2008;28(6):740-50.

46. Brandtzaeg P. Secretory IgA: Designed for Anti-Microbial Defense. Front Immunol. 2013;4:222.

47. Pyzik M, et al. FcRn: The Architect Behind the Immune and Nonimmune Functions of IgG and Albumin. J Immunol. 2015;194(10):4595-603.

48. Rogier EW, et al. Secretory antibodies in breast milk promote long-term intestinal homeostasis by regulating the gut microbiota and host gene expression. Proc Natl Acad Sci U S A. 2014;111(8):3074-9.

49. Giugliano LG, et al. Free secretory component and lactoferrin of human milk inhibit the adhesion of enterotoxigenic Escherichia coli. J Med Microbiol. 1995;42(1):3-9.

50. Corthesy B. Multi-faceted functions of secretory IgA at mucosal surfaces. Front Immunol. 2013;4:185.

51. Israel EJ, et al. Expression of the neonatal Fc receptor, FCRn, on human intestinal epithelial cells. Immunology. 1997;92(1):69-74.

52. Latvala S, et al. Distribution of FcRn Across Species and Tissues. J Histochem Cytochem. 2017;65(6):321-33.

53. Dickinson $\mathrm{BL}$, et al. Bidirectional FCRn-dependent IgG transport in a polarized human intestinal epithelial cell line. J Clin Invest. 1999;104(7):903-11.

54. Aaen $\mathrm{KH}$, et al. The neonatal Fc receptor in mucosal immune regulation. Scand $J$ Immunol. 2021;93(2):e13017.

55. Krol KM, and Grossmann T. Psychological effects of breastfeeding on children and mothers. Bundesgesundheitsblatt Gesundheitsforschung Gesundheitsschutz. 2018;61(8):977-85.

56. Spencer WJ, et al. Alpha-lactalbumin in human milk alters the proteolytic degradation of soluble CD14 by forming a complex. Pediatr Res. 2010;68(6):490-3.

57. Chatterton $\mathrm{DE}$, et al. Anti-inflammatory mechanisms of bioactive milk proteins in the intestine of newborns. Int J Biochem Cell Biol. 2013;45(8):1730-47.

58. Torner L. Actions of Prolactin in the Brain: From Physiological Adaptations to Stress and Neurogenesis to Psychopathology. Front Endocrinol (Lausanne). 2016;7:25.

59. Woo SH, et al. Osteopontin Protects Colonic Mucosa from Dextran Sodium SulfateInduced Acute Colitis in Mice by Regulating Junctional Distribution of Occludin. Dig Dis Sci. 2019;64(2):421-31.

60. Andoh $\mathrm{A}$, et al. Intestinal trefoil factor induces decay-accelerating factor expression and enhances the protective activities against complement activation in intestinal epithelial cells. J Immunol. 2001;167(7):3887-93. 
61. Puig KL, et al. Amyloid precursor protein mediated changes in intestinal epithelial phenotype in vitro. PLoS One. 2015;10(3):e0119534.

62. Tuli A, et al. Amyloid precursor-like protein 2 increases the endocytosis, instability, and turnover of the H2-K(d) MHC class I molecule. J Immunol. 2008;181(3):1978-87.

63. Austin K, et al. IGF binding protein-4 is required for the growth effects of glucagon-like peptide-2 in murine intestine. Endocrinology. 2015;156(2):429-36.

64. Tassi $E$, et al. Impact of fibroblast growth factor-binding protein-1 expression on angiogenesis and wound healing. Am J Pathol. 2011;179(5):2220-32.

65. Cabral-Pacheco GA, et al. The Roles of Matrix Metalloproteinases and Their Inhibitors in

66. Quadros EV, et al. Transcobalamin II synthesized in the intestinal villi facilitates transfer

67. Das B, et al. Enteroids expressing a disease-associated mutant of EpCAM are a model for

68. Moon R, et al. Complement C3 production in human intestinal epithelial cells is congenital tufting enteropathy. Am J Physiol Gastrointest Liver Physiol. 2019;317(5):G580-G91. regulated by interleukin 1 beta and tumor necrosis factor alpha. Arch Surg. 1997;132(12):1289-93.

69. Kopp ZA, et al. Do antimicrobial peptides and complement collaborate in the intestinal mucosa? Front Immunol. 2015;6:17.

70. Andoh A, et al. Differential cytokine regulation of complement $\mathrm{C} 3, \mathrm{C} 4$, and factor $\mathrm{B}$ synthesis in human intestinal epithelial cell line, Caco-2. J Immunol. 1993;151(8):423947.

71. Ogundele M. Role and significance of the complement system in mucosal immunity: particular reference to the human breast milk complement. Immunol Cell Biol. 2001;79(1):1-10.

72. Morrin ST, et al. Interrogation of Milk-Driven Changes to the Proteome of Intestinal Epithelial Cells by Integrated Proteomics and Glycomics. J Agric Food Chem. 2019;67(7):1902-17.

73. Sato $\mathrm{T}$, et al. Long-term expansion of epithelial organoids from human colon, adenoma, adenocarcinoma, and Barrett's epithelium. Gastroenterology. 2011;141(5):1762-72.

\section{Figure legends}

629 Figure 1. Pediatric and adult enteroid monolayers exhibit distinct maturation features. (A)

630 Confocal microscopy images (XZ projections) depicting the difference in epithelial cell height

631 between pediatric and adult enteroid monolayers. Actin, magenta; DNA, blue. Scale bar=20 $\mu \mathrm{m}$.

632 (B) Epithelial cell heights quantified by immunofluorescent confocal microscopy analysis ( $\geq 8$

633 different view fields). (C) TER values of enteroid monolayers. Images are representative of three 
634 independent experiments $(A)$. Data shown in $(B)$ and $(C)$ represent the mean \pm SEM from three

635 (B) or two (C) independent experiments that included $n=8-12$ enteroid monolayers/group per

636 experiment. Each symbol represents an independent monolayer. (A-C) All measurements

637 included 2 pediatric- and 3 adult-derived monolayers. (B, C) p-values were calculated by 638 Student's $t$ test.

640 Figure 2. Human milk decreases ion permeability of the pediatric intestinal epithelium.

641 (A) TER values of 2PD monolayers apically treated with $2 \%$ or $20 \%(\mathrm{v} / \mathrm{v})$ of human milk (HM).

642 (B) TER measurement of 2PD and 5PD monolayers apically treated with $20 \%(\mathrm{v} / \mathrm{v})$ of $\mathrm{HM}$ or $64320 \%(\mathrm{w} / \mathrm{v})$ of commercial infant formula. Mean \pm SEM. are shown. Data are representative of 644 three independent experiments with $n=3-6$ enteroid monolayers/group per experiment. $p$-values 645 were calculated by one-way-ANOVA with Šidák's post-hoc analysis. Unless indicated, p-values correspond to treated vs. non-treated controls.

648 Figure 3. Human milk modulates occludin expression. (A) Confocal microscopy images (XY 649 and $\mathrm{YZ}$ projections) of 2PD enteroid monolayers untreated (NT) or apically treated for 24h with $650 \mathrm{HM}(20 \% ; \mathrm{v} / \mathrm{v})$ or IF (20\%; w/v). Occludin, green; actin, magenta. Scale bar=10 $\mu \mathrm{m}$. (B) Relative 651 fluorescence intensity of occludin quantified by confocal microscopy analysis of 2PD (left) and 652 5PD (right) monolayers treated with HM (20\%; v/v) or IF (20\%; w/v) for $24 \mathrm{~h}$ and $72 \mathrm{~h}$. Mean \pm 653 SEM are shown. Data are pooled from three independents with $n=4-6$ enteroid 654 monolayers/group per experiment. Each symbol indicates an independent monolayer. p-values 655 were calculated by one-way-ANOVA with Šidák's post-hoc analysis. (C) Confocal microscopy 656 images (XY projections) of 5PD enteroid monolayers treated with HM for 48h. Occludin, green; 657 lysozyme (Lyz; XY projection), red; trefoil factor 3 (TFF3; XY projection), red; chromogranin A 658 (ChgA; XY and XZ projections), red; actin, magenta; DNA, blue. Paneth and goblet cells, scale 
659 bar=5 $\mu \mathrm{m}$; enteroendocrine cells, scale bar=10 $\mu \mathrm{m}$. (A and C) Data are representative of three

660 independent experiments with $n=3$ enteroid monolayers/group per experiment.

662 Figure 4. Human milk modulates epithelial innate immune mediators. (A) Relative

663 fluorescence intensity of human DEFA5 quantified by confocal microscopy analysis of 2PD and 664 5PD monolayers NT or treated with HM (20\%; v/v) or IF (20\%; w/v) for 48h. (B) Representative 665 confocal microscopy images (XY projections) of 5PD monolayer showing localization 666 (arrowheads) of DEFA5 in Lyz cells in HM-treated monolayer. DEFA5, green; Lyz, red; actin,

667 magenta; DNA, blue. Scale bar=10 $\mu \mathrm{m}$. (C) Representative confocal microscopy images (XY 668 projections) of 5PD monolayer depicting co-localization (arrowheads) of TFF3 (red) and DEFA5 669 (green); DNA, blue. Scale bar=50 $\mu \mathrm{m}$. (D-F) Total amount of MCP-1, GM-CSF, and IL-8 in the 670 apical media of 2PD monolayer treated as described in (A) for $24 \mathrm{~h}$ and $72 \mathrm{~h}$. (G) PCA plot from 671 HM-, and IF-treated, and NT enteroid monolayers for 24h. PC, principal component. Variables 672 analyzed: TER, occludin, DEFA5, MCP-1, GM-CSF, IL-8. (A, D-F). Mean \pm SEM are shown.

673 Data are representative of three independent experiments with $n=6-12$ enteroid 674 monolayers/group per experiment. Each symbol indicates an independent monolayer. p-values 675 were calculated by one-way-ANOVA with Tukey's post-test for multiple comparisons.

677 Figure 5. Breast milk enhances expression of plgR and slgA translocation across the 678 epithelial monolayers. (A) Confocal microscopy images showing SC (left, XZ projections, 679 scale bar=10 $\mu \mathrm{m}$; right, $\mathrm{XY}$ projection, scale bar=5 $\mu \mathrm{m}$ ) in 5PD enteroid monolayer NT or treated 680 with $20 \%(\mathrm{v} / \mathrm{v})$ of HM for $72 \mathrm{~h}$. SC, green; actin, red; DNA, blue. (B) Composite immunoblotting 681 (IB) showing SC and FcRn expression in non-treated 2PD and 5PD monolayers. (C) IB showing 682 plgR expression in HM and IF, and 2PD monolayers NT or treated with $20 \%(\mathrm{v} / \mathrm{v}) \mathrm{HM}$. MW, 683 molecular weight. (D) Total $\lg A$ and $\lg G$ in the basolateral media of pediatric monolayers treated 
684 for $48 \mathrm{~h}$ with $20 \%$ (v/v) HM. Data represent mean \pm SEM of three combined experiments, each

685 including 2 monolayers/group per experiment. Each symbol indicates an independent

686 monolayer. p-value was calculated by Student's $t$ test.

688 Figure 6. Human milk modifies epithelial cell protein expression and basolateral

689 secretion. (A) Volcano plot of differential protein abundance (high false discovery rate) in the

690 basolateral culture supernatant of 2PD monolayers NT $(n=2)$ or treated with $20 \% \mathrm{HM}(\mathrm{v} / \mathrm{v})(n=3)$

691 for 24h. Red dots indicate HM unique proteins; blue dots indicate epithelial cell-derived proteins;

692 green dots indicate proteins derived from both $\mathrm{HM}$ and epithelial cells. (B) Protein-protein

693 interaction analysis of 61 upregulated proteins produced by HM-treated monolayers selected

694 based on the cut off shown in $(A)$. Medium confidence interaction score $=0.400$. A thicker line

695 between nodes indicates stronger protein-protein interaction. (C) Enrichment analysis of GO

696 terms annotated for cellular component, molecular function, and biological process of the 61

697 upregulated proteins as described in (A). 


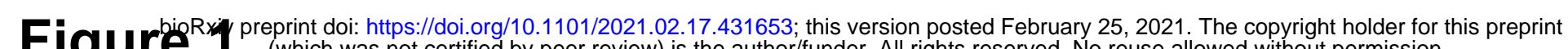
10 (which was not certified by peer review) is the author/funder. All rights reserved. No reuse allowed without permission.

A Pediatric

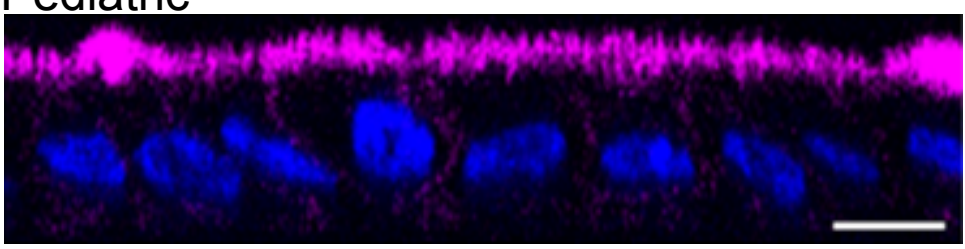

\section{Adult}

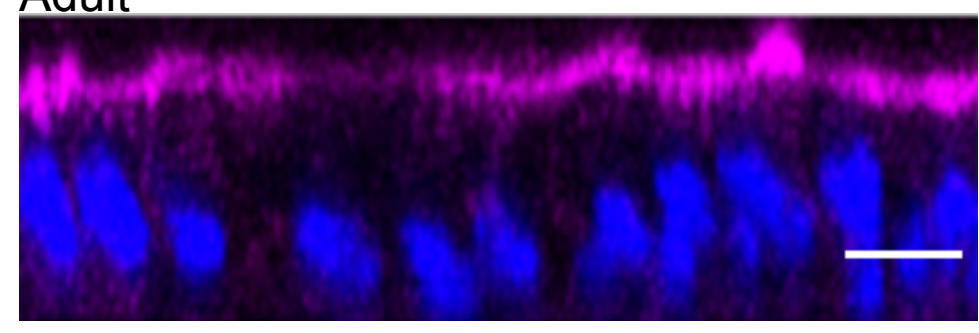

\section{B}

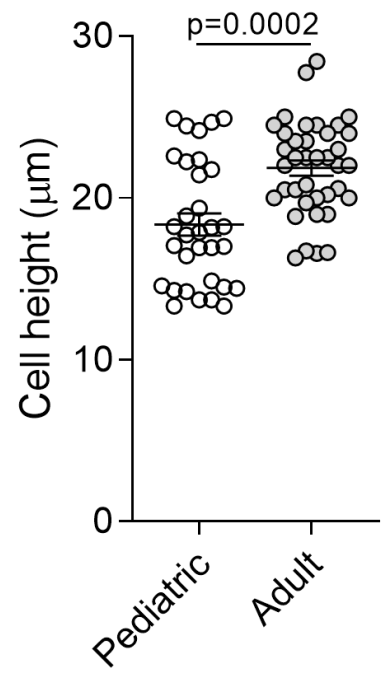

C

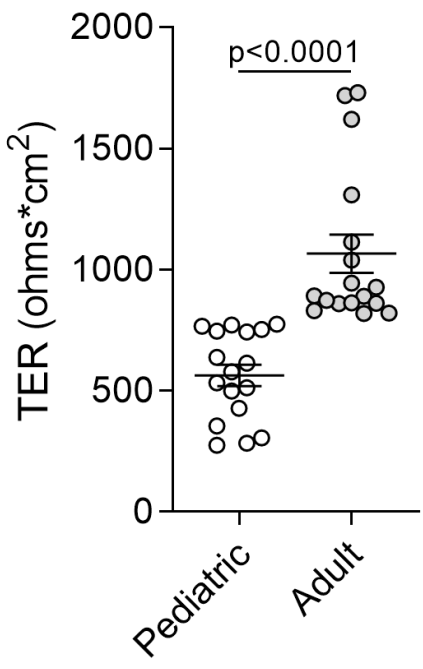


Figure 3
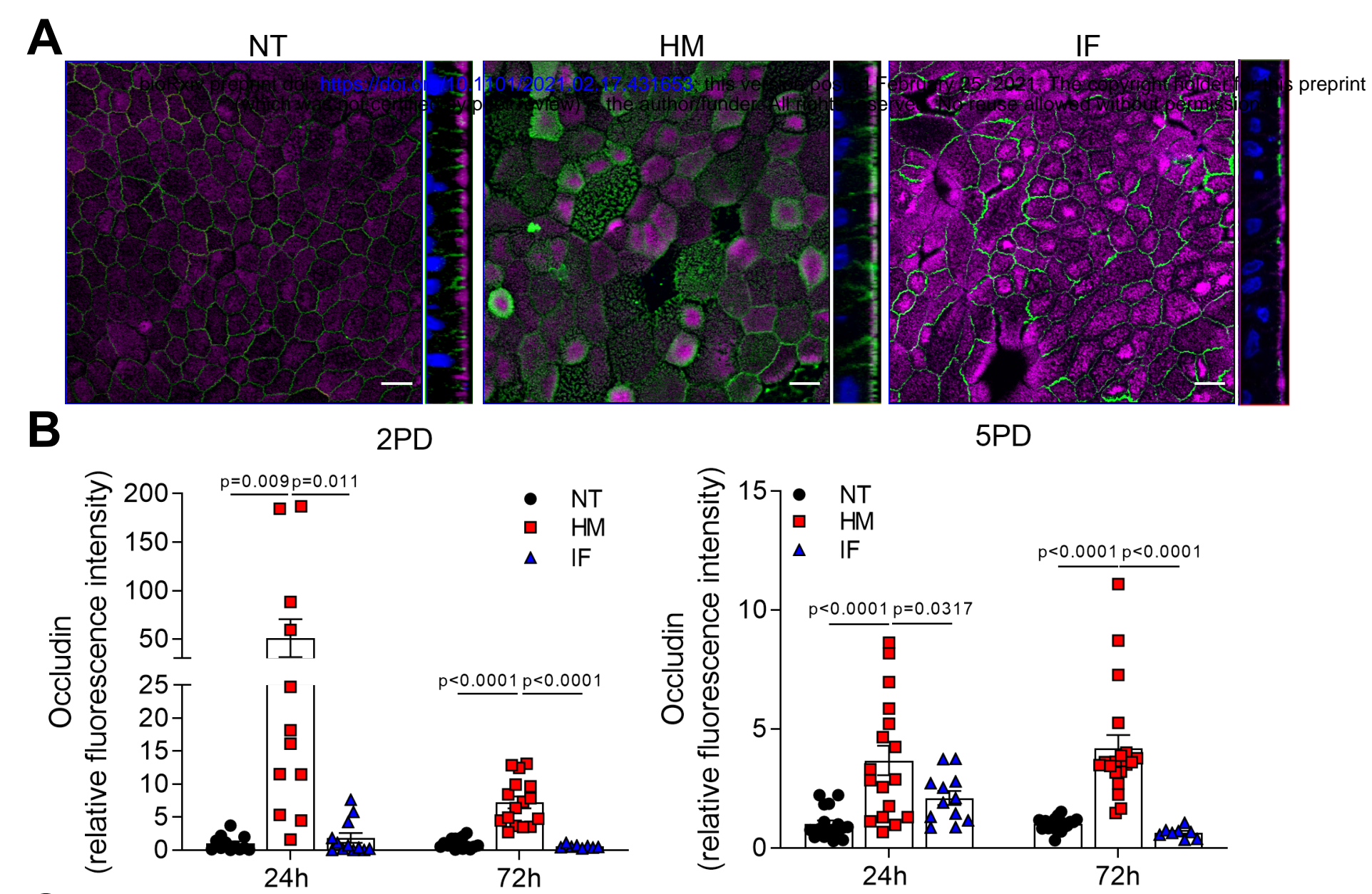

C

Paneth cells

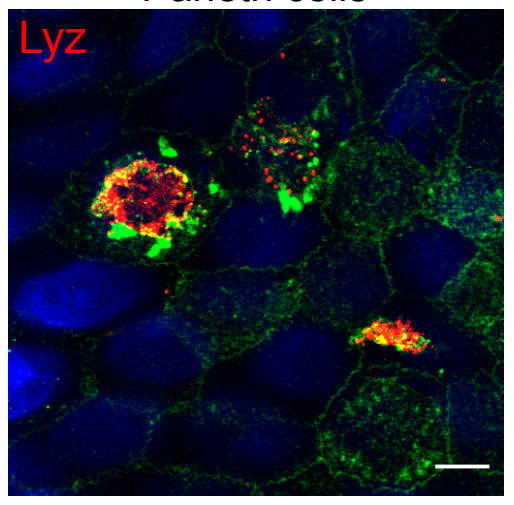

Goblet cells

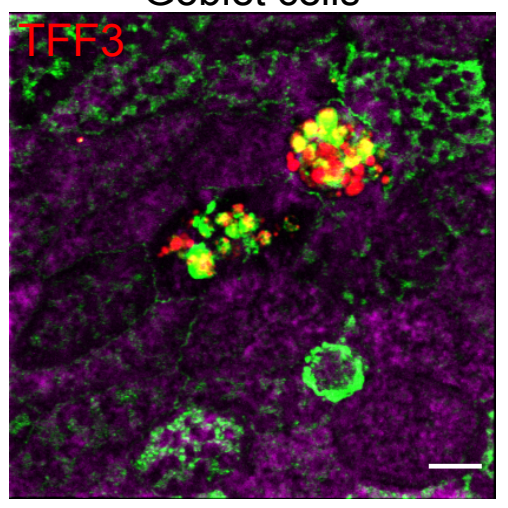

Enteroendocrine cells

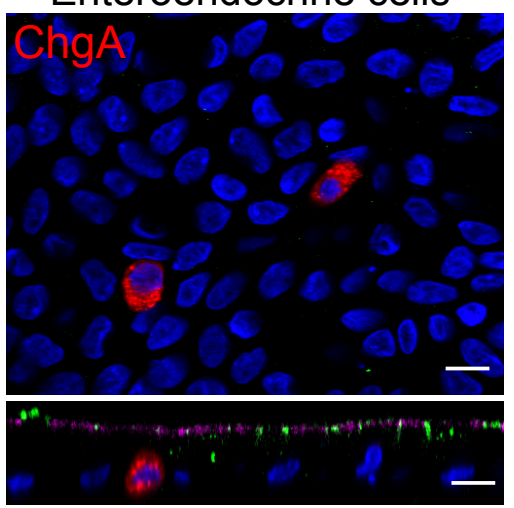


Figure 4

A

B

NT

HM

IF
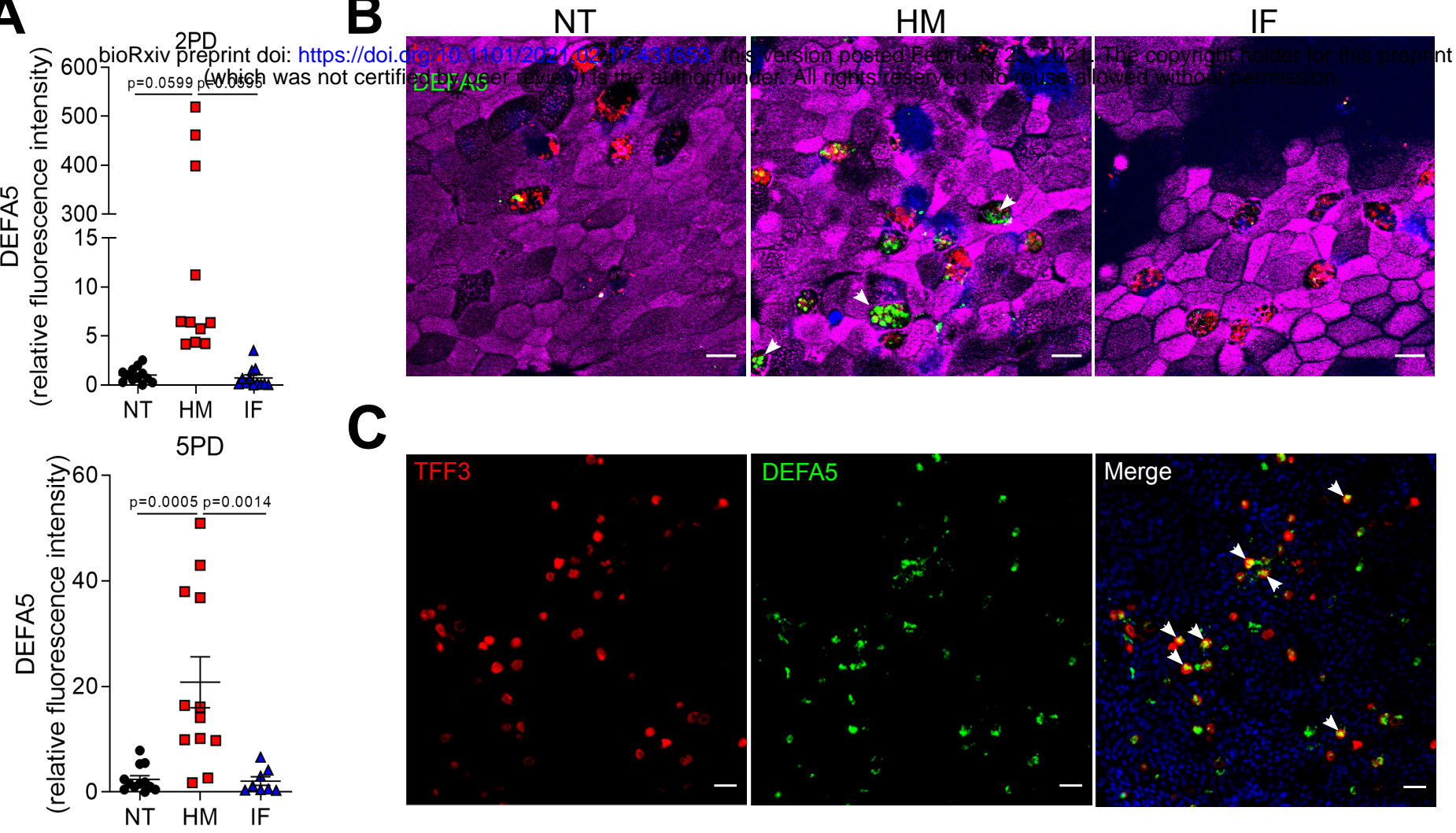

D
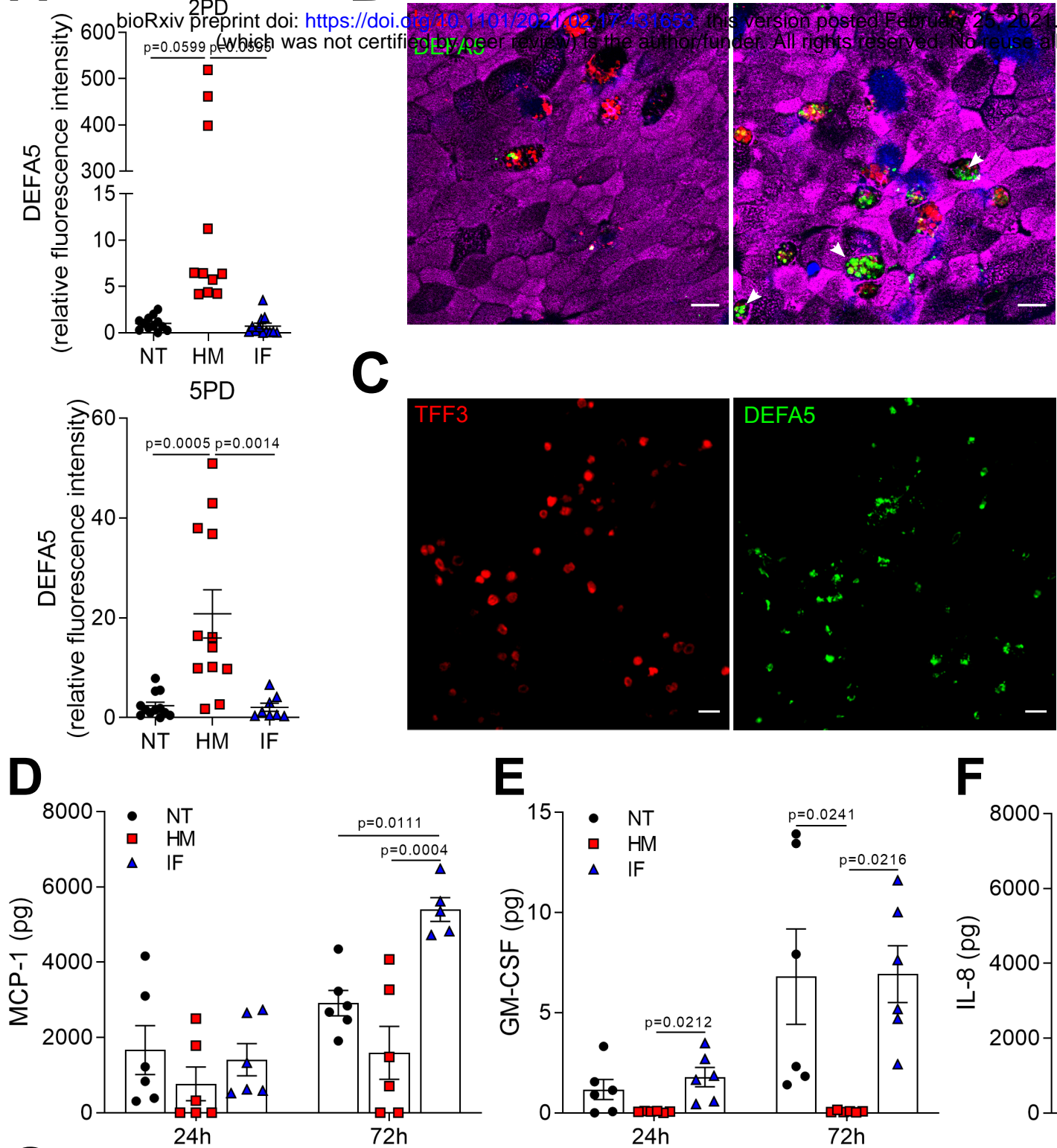

F

G

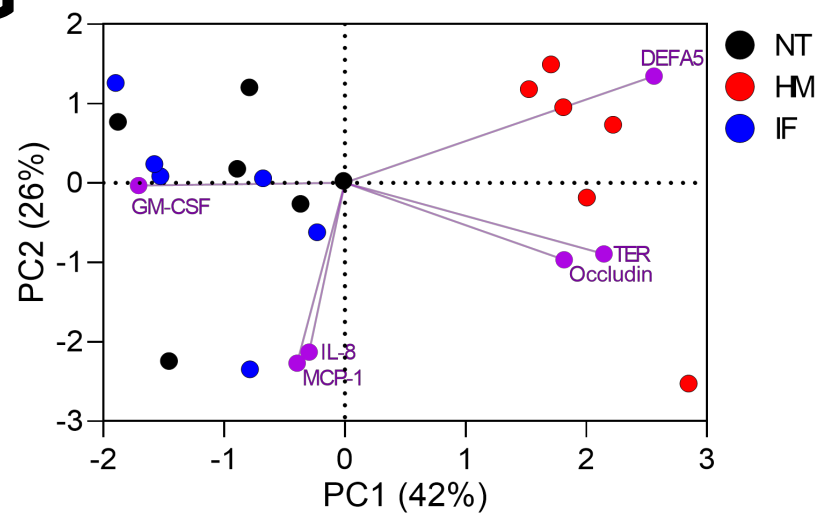




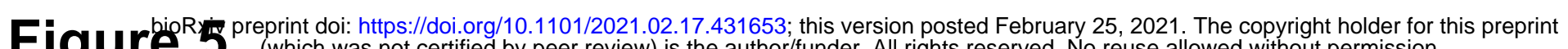

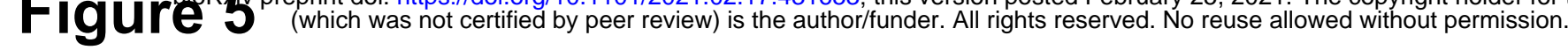
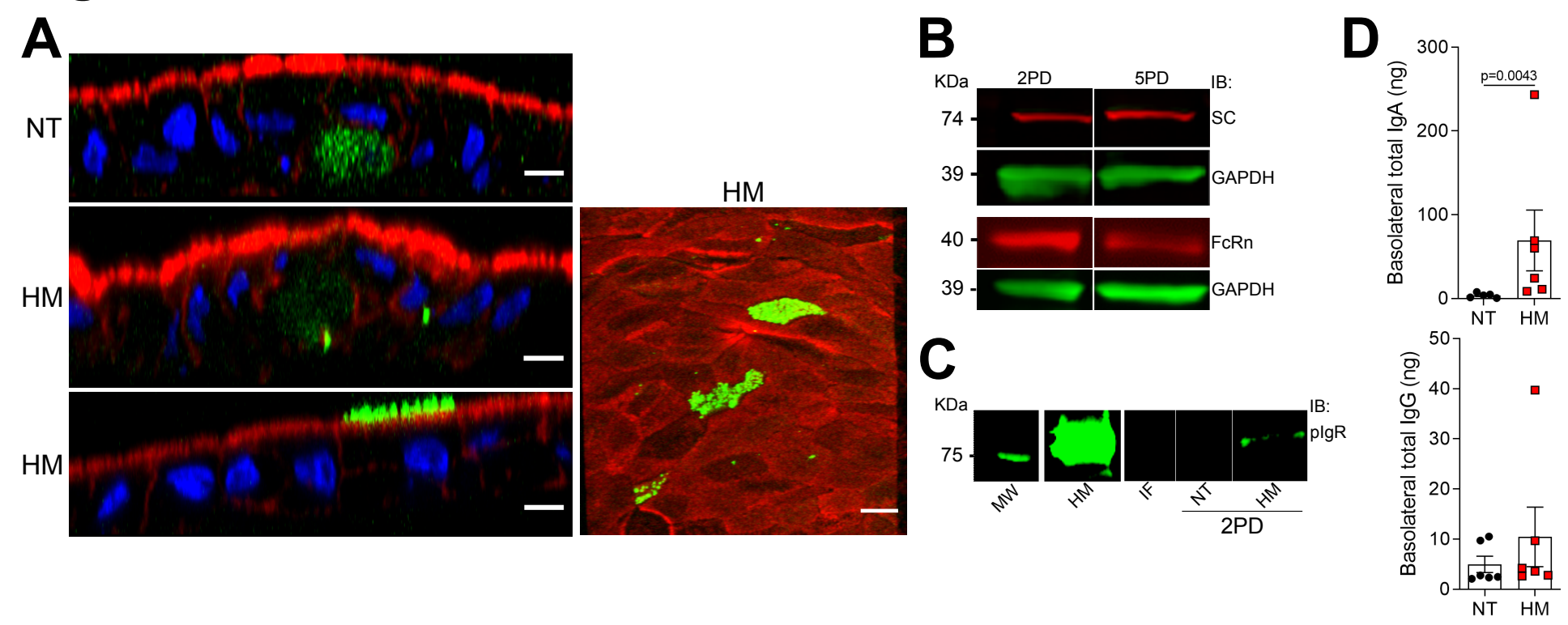

FF Fure
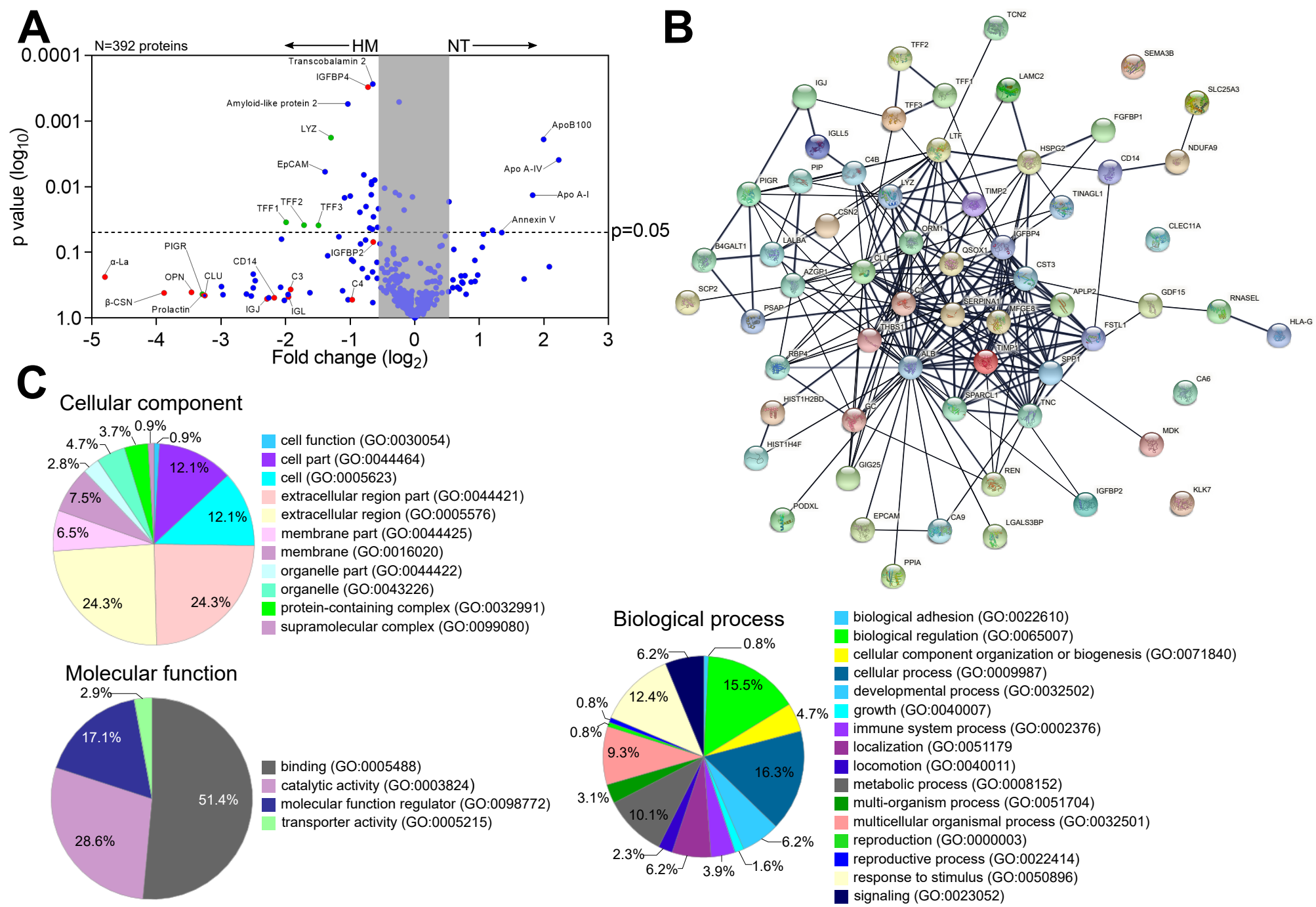

binding (GO:0005488)

catalytic activity (GO:0003824)

molecular function regulator (GO:0098772)

transporter activity (GO:0005215)

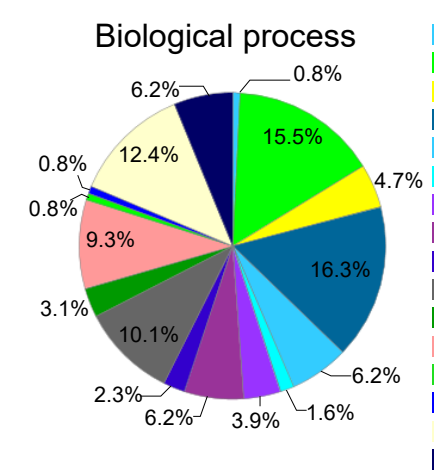

biological adhesion (GO:0022610)

biological regulation (GO:0065007)

cellular component organization or biogenesis (GO:0071840)

cellular process (GO:0009987)

developmental process (GO:0032502)

growth (GO:0040007)

immune system process (GO:0002376)

localization (GO:0051179

locomotion (GO:0040011)

metabolic process (GO:0008152)

multi-organism process (GO:0051704)

multicellular organismal process (GO:0032501)

reproduction (GO:0000003)

reproductive process (GO:0022414)

response to stimulus (GO:0050896)

signaling (GO:0023052) 